COMMENT. Convulsion with mild gastroenteritis $(\mathrm{CwG})$ is a frequent seizure disorder in Japan (Uemura $N$ et al. Brain Dev 2002;24:745-9). Previously described as generalized, the seizures appear to be partial with secondary generalization. CwG presents in previously healthy infants or young children aged 6 months to 3 years. Seizures are afebrile and occur in clusters in close relation to the onset of diarrhea. Laboratory results including electrolytes, glucose, and CSF are normal, ictal EEG is focal and secondary generalized, the interictal EEG is normal, and the outcome benign. In the above study, no reports of neuroimaging are included. Rotavirus was the likely cause in 2 of the 6 patients.

Usually affecting Japanese children, CwG is now known to occur outside Asia. A study of 14 children with the syndrome is reported from the UK (Narchi H. Benign afebrile cluster convulsions with gastroenteritis. BMC Pediatr 2004 Feb 5;4:2 [abstract]). The gastroenteritis was considered viral, and Rotavirus was detected by enzyme immunoassay in the stools of 7 of 10 patients tested. Stool culture was negative for pathogenic bacteria. CSF and computed tomography, performed on 2 patients with prolonged seizures, were negative. On long-term follow-up, none had recurrence of seizures and all had normal development. The seizure mechanism is unknown, and CNS complications are not generally described with Rotavirus infection (AAP Redbook). In the absence of fever, electrolyte and glucose abnormalities, a viral neurotropism, similar to that suggested for HHV-6 in febrile seizures (Hall CB et al, 1994; Millichap JG and JJ, 2006), might be considered.

\title{
INFECTIOUS MONONUCLEOSIS AND MULTIPLE SCLEROSIS
}

A cohort of 25,234 Danish patients testing positive (at titers of 1/32) for Epstein-Barr virus related mononucleosis (MONO), during the period 1940-1988, was followed for more than 550,000 person-years. The cohort was linked to the Multiple Sclerosis (MS) Registry for determination of risk of developing MS, in a study at Statens Serum Institut, Copenhagen. A total of 104 cases of MS observed vs 46 expected in the cohort testing positive for MONO corresponded to a standardized incidence ratio (SIR) of 2.27. In a cohort with negative MONO test, 68 observed cases corresponded to a SIR of 1.23, and similar to that prevailing in the general population. Elevated SIRs for MS were observed for MONO patients at all ages, except those 30 years or older. SIR for MS diagnosis was inversely correlated with age at MONO infection ( $\mathrm{P}$ for trend $=0.03$ ). Increased risk of MS appeared 10 years or more after MONO occurred and remained increased for more than 3 decades. The risk was uniformly distributed for sex and age; it was not correlated with severity of MONO infection. (Nielsen TR, Rostgaard K, Nielsen NM, et al. Multiple sclerosis after infectious mononucleosis. Arch Neurol Jan 2006;64:72-75). (Respond: TR Nielsen MD, Department of Epidemiology Research, Statens Serum Institut, Artillerivej 5, DK-2300 Copenhagen S, Denmark).

COMMENT. After recovery from infectious mononucleosis, a patient has a greater than 2-fold increased risk of developing multiple sclerosis. The risk of MS is increased soon after mononucleosis and extends for up to 30 years; it is independent of sex, age, and time from infection or severity of infection.

The association of 3-methylcrotonyl-CoA carboxylase deficiency and severe multiple sclerosis is reported in a 13-year-old female (Darin $\mathrm{N}$ et al. Pediatr Neurol Feb 2007;36:132-134). She presented with a mild Reye-like syndrome following influenza A 
infection at age 5 years. Alopecia areata, psychomotor retardation and ADHD followed, and the diagnosis of MCC deficiency was made at the onset of multiple sclerosis. Urinary organic acids showed an increased excretion of 3-MCG and 3-HIVA, carnitine was decreased, and enzyme measurements in cultured fibroblasts confirmed the 3-MCC deficiency. Response to interferon was poor, but a good response followed treatment with mitoxantrone.

\section{CHRONIC INFLAMMATORY POLYNEUROPATHY}

Thirteen children with chronic inflammatory demyelinating polyneuropathy monitored between 1975 and 2005 are reported from Centre hospitalier universitaire SainteJustine, Montreal, Canada. Age at diagnosis varied between 3 and 14 years; 4 were female and 9 male. All presented with symmetrical muscle weakness, mainly in the lower limbs. One had asymmetrical upper limb weakness. Symptoms evolved over 1 week to 1 year, and diagnosis was confirmed after 4 weeks of progression or relapsing course. Sensory symptoms, hypoesthesia and painful paresthesias, occurred in $54 \%(7 / 13)$ of patients at diagnosis. Two patients had diplopia. A respiratory tract viral infection preceded onset in $23 \%(3 / 13)$. Disability varied between minor and severe, 2 to 5 on the Rankin score. Diagnosis was confirmed by delayed nerve conduction, elevated protein $>35 \mathrm{mg} / \mathrm{dL}$ and leukocytes $<10 / \mathrm{mm}^{3}$ in the CSF, and nerve biopsy in 5 patients $(38 \%)$, showing inflammation and segmental demyelination. Evolution was polyphasic in $10(77 \%)$ patients, with 1 to 10 relapses over a follow-up of 6 months to 5 years. Patients treated before 2000 (7/13) received prednisone with good response. Some required repeat courses at relapse and some received azathioprine or cyclophosphamide in addition. After 2000, 3 of 4 patients received intravenous immunoglobulin with an excellent initial response. All required repeat treatments for relapses, including add-on therapies in 2. Two recent patients are refractory, but their disabilities are only minimal to minor. (Rossignol E, D'Anjou G, Lapointe N, Haddad E, Vanasse M. Evolution and treatment of childhood chronic inflammatory polyneuropathy. Pediatr Neurol Feb 2007;36:88-94). (Respond: Dr Vanasse, Service de neurologie, 5 Bloc 4, Centre hospitalier universitaire Ste-Justine, 3175 Cote Ste-Catherine, Montreal, Quebec, Canada H3T 1C5).

COMMENT. Chronic inflammatory demyelinating polyneuropathy (CIDP) is an acquired progressive symmetrical proximal weakness with areflexia, associated with paresthesias or ataxia. Presentation in children is more subacute than chronic, with numerous relapses. Prognosis is usually favorable with complete functional recovery. In the above study, most had relapses requiring repeat courses of prednisone and add-on therapies, and some cases were refractory to treatment.

In a report of 39 patients with CIDP treated with either high-dose, intermittent IV methylprednisolone (IV MP), IV immunoglobulin (IVIg) or oral prednisone or cyclosporine, 81 to $88 \%$ showed improvement in muscle strength, and these treatments were equally effective. Weight gain and cushingoid features were less frequent in patients treated with IVMP (19\% affected) than in those receiving oral prednisone (58\%). IVMP as initial and maintenance therapy is recommended in CIDP patients with weakness or disability (Lopate $G$ et al. Arch Neurol 2005;62:249-254; Ped Neur Briefs March 2005;19:18). 